\title{
NOTE ON THE STRESSES PRODUCED BY NUCLEI OF THERMO-ELASTIC STRAIN IN A SEMI-INFINITE ELASTIC SOLID*
}

By BIBHUTIBHUSAN SEN (Presidency College, Calcutta)

1. Introduction. It has been shown by Goodier ${ }^{1}$ that if an element of volume $d \Omega$ surrounding a point $C$ in an infinite elastic solid is at a temperature $T$ while the rest of the solid is at zero temperature, the displacement at any point is the gradient of a function

$$
-\frac{\alpha(1+\nu)}{(1-\nu)} \cdot \frac{T}{4 \pi} \cdot \frac{d \Omega}{r}
$$

where $\nu$ is Poisson's ratio, $\alpha$, the coefficient of linear expansion and $r$, the distance of the point from $C$. The singularity at $C$ is the simple 'centre of dilatation' which is supposed to form a nucleus of thermo-elastic strain at the point. The problem solved in this paper is that of determining displacements and stresses in an isotropic semi-infinite elastic solid which has a nucleus of thermo-elastic strain as defined above at any point $C$ below the plane boundary.

2. Statement of the problem. We suppose that the solid is bounded by the plane $Z=0$, the axis of $Z$ being drawn into the body. The coordinates of $C$ are taken as $(0,0, c)$. Putting

$$
P=-\frac{\alpha(1+\nu)}{(1-\nu)} \cdot \frac{T d \Omega}{4 \pi}
$$

and

$$
V_{1}=\frac{1}{R_{1}}=\frac{1}{\left[x^{2}+y^{2}+(z-c)^{2}\right]^{1 / 2}}
$$

we can express the components of displacement $u^{\prime}, v^{\prime}, w^{\prime}$ due to the nucleus of thermoelastic strain situated at $C$ as

$$
u^{\prime}=P \frac{\partial v_{1}}{\partial x}, \quad v^{\prime}=P \frac{\partial v_{1}}{\partial y}, \quad w^{\prime}=P \frac{\partial v_{1}}{\partial z} .
$$

These components give us the stresses

$$
\begin{aligned}
\tau_{x z}^{\prime} & =\frac{E P}{(1+\nu)} \frac{\partial^{2} v_{1}}{\partial x \partial z}=\frac{3 E P}{(1+\nu)} \frac{x(z-c)}{R_{1}^{5}}, \\
\tau_{y z}^{\prime} & =\frac{E P}{(1+\nu)} \frac{\partial^{2} v_{1}}{\partial y \partial z}=\frac{3 E P}{(1+\nu)} \frac{y(z-c)}{R_{1}^{5}}, \\
\sigma_{z}^{\prime} & =\frac{E P}{(1+\nu)} \frac{\partial^{2} v_{1}}{\partial z^{2}}=\frac{E P}{(1+\nu)}\left[-\frac{1}{R_{1}^{3}}+\frac{3(z-c)^{2}}{R_{1}^{5}}\right],
\end{aligned}
$$

$E$ being the Young's modulus.

${ }^{*}$ Received Dec. 19, 1949.

${ }^{1} \mathrm{~J}$. N. Goodier, On the integration of thermo-elastic equations, Phil. Mag. 23, 1017 (1937). 
In order that the surface $Z=0$ may be free from stress, we must superimpose a stress system $\tau_{x z}^{\prime \prime}, \tau_{v z}^{\prime \prime}, \sigma_{z}^{\prime \prime}$ possible in the body such that

$$
\begin{aligned}
& {\left[\tau_{x z}^{\prime \prime}\right]_{z=0}=-\left[\tau_{x z}^{\prime}\right]_{z=0}=\frac{3 E P x c}{(1+\nu)\left(x^{2}+y^{2}+c^{2}\right)^{5 / 2}},} \\
& {\left[\tau_{y z}^{\prime \prime}\right]_{z=0}=-\left[\tau_{y z}^{\prime}\right]_{z=0}=\frac{3 E P y c}{(1+\nu)\left(x^{2}+y^{2}+c^{2}\right)^{5 / 2}},} \\
& {\left[\sigma_{z}^{\prime \prime}\right]_{z=0}=-\left[\sigma_{z}^{\prime}\right]_{z=0}=\frac{E P}{(1+\nu)}\left[\frac{1}{\left(x^{2}+y^{2}+c^{2}\right)^{3 / 2}}-\frac{3 c^{2}}{\left(x^{2}+y^{2}+c^{2}\right)^{5 / 2}}\right] .}
\end{aligned}
$$

These stresses $\tau_{x z}^{\prime \prime}, \tau_{y z}^{\prime \prime}, \sigma_{z}^{\prime \prime}$ can be obtained easily by the following method which was given by the author in a previous paper. ${ }^{2}$

3. Solution. Since by assumption $\tau_{x z}^{\prime \prime}, \tau_{y z}^{\prime \prime}, \sigma_{z}^{\prime \prime}$ are stresses possible in an isotropic elastic solid, they must satisfy the equations

$$
\begin{aligned}
& \nabla^{2} \tau_{x z}^{\prime \prime}+\frac{1}{(1+\nu)} \frac{\partial^{2} \theta_{2}}{\partial x \partial z}=0, \\
& \nabla^{2} \tau_{\nu z}^{\prime \prime}+\frac{1}{(1+\nu)} \frac{\partial^{2} \theta_{2}}{\partial y \partial z}=0, \\
& \nabla^{2} \sigma_{z}^{\prime \prime}+\frac{1}{(1+\nu)} \frac{\partial^{2} \theta_{2}}{\partial z^{2}}=0,
\end{aligned}
$$

where $\nabla^{2}$ is the Laplace operator, and $\theta_{2}=\sigma_{x}^{\prime \prime}+\sigma_{y}^{\prime \prime}+\sigma_{z}^{\prime \prime} . \theta_{2}$ being a harmonic function, we can obtain the solutions of the above equations as

$$
\begin{aligned}
& \tau_{x z}^{\prime \prime}=-\frac{1}{2(1+\nu)} z \frac{\partial \theta_{2}}{\partial x}+\phi_{1}, \\
& \tau_{y z}^{\prime \prime}=-\frac{1}{2(1+\nu)} z \frac{\partial \theta_{2}}{\partial y}+\phi_{2}, \\
& \sigma_{z}^{\prime \prime}=-\frac{1}{2(1+\nu)} z \frac{\partial \theta_{2}}{\partial z}+\phi_{3},
\end{aligned}
$$

where $\phi_{1}, \phi_{2}$ and $\phi_{3}$ are harmonic functions. Using the results (6) and (8) we find that

$$
\begin{aligned}
& {\left[\phi_{1}\right]_{z=0}=\left[\tau_{x z}^{\prime \prime}\right]_{z=0}=\frac{3 E P x c}{(1+\nu)\left(x^{2}+y^{2}+c^{2}\right)^{5 / 2}},} \\
& {\left[\phi_{2}\right]_{z=0}=\left[\tau_{y z}^{\prime \prime}\right]_{z=0}=\frac{3 E P y c}{(1+\nu)\left(x^{2}+y^{2}+c^{2}\right)^{5 / 2}},} \\
& {\left[\phi_{3}\right]_{z=0}=\left[\sigma_{z}^{\prime \prime}\right]_{z=0}=\frac{E P}{(1+\nu)}\left[\frac{1}{\left(x^{2}+y^{2}+c^{2}\right)^{3 / 2}}-\frac{3 c^{2}}{\left(x^{2}+y^{2}+c^{2}\right)^{5 / 2}}\right] .}
\end{aligned}
$$

'B. Sen, Stresses due to forces and couples acting in the interior of a semi-infinite elastic solid, Bull. Cal. Math. Soc. 32, 73-83 (1940). 
Putting

$$
V_{2}=\frac{1}{R_{2}}=\frac{1}{\left[x^{2}+y^{2}+(z+c)^{2}\right]^{1 / 2}},
$$

we find that the conditions (9) are satisfied if we write

$$
\phi_{1}=\frac{E P}{(1+\nu)} \frac{\partial^{2} v_{2}}{\partial x \partial z}, \quad \phi_{2}=\frac{E P}{(1+\nu)} \frac{\partial^{2} v_{2}}{\partial y \partial z}, \quad \phi_{3}=-\frac{E P}{(1+\nu)} \frac{\partial^{2} v_{2}}{\partial z^{2}}
$$

Since

$$
\frac{\partial \tau_{x z}^{\prime \prime}}{\partial x}+\frac{\partial \tau_{y z}^{\prime \prime}}{\partial y}+\frac{\partial \sigma_{z}^{\prime \prime}}{\partial z}=0,
$$

and $v_{2}$ is a harmonic function, we have from (8)

$$
\frac{\partial \theta_{2}}{\partial z}=2(1+\nu)\left[\frac{\partial \phi_{1}}{\partial x}+\frac{\partial \phi_{2}}{\partial y}+\frac{\partial \phi_{3}}{\partial z}\right]=-4 E P \frac{\partial^{3} v_{2}}{\partial z^{3}},
$$

whence we obtain by integration

$$
\theta_{2}=-4 E P \frac{\partial^{2} v_{2}}{\partial z^{2}}
$$

The function of integration in this case as well as in the cases that will follow, will be zero since the stresses and components of displacement must tend to zero as $Z$ tends to infinity.

Substituting into (8), the values of $\phi_{1}, \phi_{2}, \phi_{3}$ and $\theta_{2}$ obtained in (11) and (12) respectively, we have

$$
\begin{aligned}
\tau_{x z}^{\prime \prime} & =\frac{E P}{(1+\nu)}\left[2 z \frac{\partial^{3} v_{2}}{\partial x \partial z^{2}}+\frac{\partial^{2} v_{2}}{\partial x \partial z}\right], \\
\tau_{y z}^{\prime \prime} & =\frac{E P}{(1+\nu)}\left[2 z \frac{\partial^{3} v_{2}}{\partial y \partial z^{2}}+\frac{\partial^{2} v_{2}}{\partial y \partial z}\right], \\
\sigma_{z}^{\prime \prime} & =\frac{E P}{(1+\nu)}\left[2 z \frac{\partial^{3} v_{2}}{\partial z^{3}}-\frac{\partial^{2} v_{2}}{\partial z^{2}}\right] .
\end{aligned}
$$

Combining these results with those given in (5), we get

$$
\begin{aligned}
& \tau_{x z}=\tau_{x z}^{\prime}+\tau_{x z}^{\prime \prime}=\frac{E P}{(1+\nu)}\left[\frac{\partial^{2} v_{1}}{\partial x \partial z}+2 z \frac{\partial^{3} v_{2}}{\partial x \partial z^{2}}+\frac{\partial^{2} v_{2}}{\partial x \partial z}\right], \\
& \tau_{y z}=\tau_{y z}^{\prime}+\tau_{y z}^{\prime \prime}=\frac{E P}{(1+\nu)}\left[\frac{\partial^{2} v_{1}}{\partial y \partial z}+2 z \frac{\partial^{3} v_{2}}{\partial y \partial z^{2}}+\frac{\partial^{2} v_{2}}{\partial y \partial z}\right], \\
& \sigma_{\iota}=\sigma_{z}^{\prime}+\sigma_{z}^{\prime \prime}=\frac{E P}{(1+\nu)}\left[\frac{\partial^{2} v_{1}}{\partial z^{2}}+2 z \frac{\partial^{3} v_{2}}{\partial z^{3}}-\frac{\partial^{2} v_{2}}{\partial z^{2}}\right] .
\end{aligned}
$$

If $\sigma_{x}^{\prime}, \sigma_{y}^{\prime}, \sigma_{z}^{\prime}$ be calculated from $u^{\prime}, v^{\prime}, w^{\prime}$ given in (4), it will be found that

$$
\theta_{1}=\sigma_{x}^{\prime}+\sigma_{\nu}^{\prime}+\sigma_{z}^{\prime}=0 \text {. }
$$


Hence

$$
\theta=\theta_{1}+\theta_{2}=\theta_{2}=-4 E P \frac{\partial^{2} v_{2}}{\partial z^{2}} .
$$

Now let $u, v, w$ be the components of the resultant displacement parallel to the axes of $x, y$ and $z$ due to the two systems of stresses with one and two dashes respectively. Since

$$
\frac{\partial w}{\partial z}=\frac{1}{E}\left[(1+\nu) \sigma_{z}-\nu \theta\right]=P\left[\frac{\partial^{2} v_{1}}{\partial z^{2}}+2 z \frac{\partial^{3} v_{2}}{\partial z^{3}}-\frac{\dot{\partial}^{2} v_{2}}{\partial z^{2}}+4 \nu \frac{\partial^{2} v_{2}}{\partial z^{2}}\right],
$$

we find by integration

$$
\begin{aligned}
w & =P\left[\frac{\partial v_{1}}{\partial z}+2 z \frac{\partial^{2} v_{2}}{\partial z^{2}}-(3-4 \nu) \frac{\partial v_{2}}{\partial z}\right] \\
& =\frac{\alpha T d \Omega}{4 \pi} \frac{(1+\nu)}{(1-\nu)} \cdot\left[\frac{z-c}{R_{1}^{3}}+\frac{4 \nu(z+c)-(z+3 c)}{R_{2}^{3}}-\frac{6 z(z+c)^{2}}{R_{2}^{5}}\right] .
\end{aligned}
$$

Again

$$
\begin{aligned}
\frac{\partial u}{\partial z} & =\frac{2(1+\nu)}{E} \tau_{x z}-\frac{\partial w}{\partial x} \\
& =P\left[\frac{\partial^{2} v_{1}}{\partial x \partial z}+2 z \frac{\partial^{3} v_{2}}{\partial x \partial z^{2}}+2 \frac{\partial^{2} v_{2}}{\partial x \partial z}+(3-4 \nu) \frac{\partial^{2} v_{2}}{\partial x \partial z}\right]
\end{aligned}
$$

By integration we get

$$
\begin{aligned}
u & =P\left[\frac{\partial v_{1}}{\partial x}+2 z \frac{\partial^{2} v_{2}}{\partial x \partial z}+(3-4 \nu) \frac{\partial v_{2}}{\partial x}\right] \\
& =\frac{\alpha T d \Omega}{4 \pi} \frac{(1+\nu)}{(1-\nu)} x\left[\frac{1}{R_{1}^{3}}+\frac{(3-4 \nu)}{R_{2}^{3}}-\frac{6 z(z+c)}{R_{2}^{5}}\right] .
\end{aligned}
$$

Similarly from the relation

$$
\frac{\partial v}{\partial z}=\frac{2(1+\nu)}{E} \tau_{y z}-\frac{\partial w}{\partial y}
$$

we have

$$
v=\frac{\alpha T d \Omega}{4 \pi} \frac{(1+\nu)}{(1-\nu)} y\left[\frac{1}{\bar{R}_{1}^{3}}+\frac{(3-4 \nu)}{R_{2}^{3}}-\frac{6 z(z+c)}{R_{2}^{5}}\right] .
$$

Components of displacement being given by (16), (17) and (18), the stress components (other than $\tau_{x z}, \tau_{y z}, \sigma_{z}$ which have already been obtained in (14)) can be easily calculated.

We find that on the plane $Z=0$.

$$
[w]_{z=0}=-\frac{\alpha T d \Omega}{\pi} \frac{(1+\nu) c}{R_{0}^{3}},
$$


where

$$
R_{0}^{2}=x^{2}+y^{2}+c^{2} .
$$

It is evident that if there be a continuous distribution of such nuclei (as enunciated above) over a certain region, we can find the total effect by integration over the region.

\section{PRACTICAL SOLUTION OF SIMULTANEOUS LINEAR EQUATIONS*}

By O. L. BOWIE (Watertown Arsenal, Watertown, Mass.)

I. Introduction. The effect of "rounding off" errors in the solution of the set of simultaneous linear equations with constant coefficients

$$
\sum_{i=1}^{n} a_{i j} x_{i}=c_{i} \quad(i=1,2, \cdots, n)
$$

can lead to considerable inaccuracy of solution. Furthermore, the accuracy of an approximate solution of (1) usually cannot be determined from the errors in the individual equations without an excessive amount of additional computation. Unfortunately, most conventional techniques lead unavoidably to "rounding off" errors and the computer is often faced with both of the above problems.

When the form of (1) is such that the well-known" "Classical Iterative" technique can be applied, the difficulties above are usually not encountered. In most practical problems the coefficients and the desired solution can be handled well within the digital capacity of the average desk calculator. Since only the coefficients and approximations of the solution occur in the process, the necessity of "rounding off" is therefore usually eliminated. Accurate estimation of the accuracy of an approximate solution is inherent in the iterative character of the process.

Although rigorous investigations have been made as to the form of (1) sufficient to ensure convergence with the Iterative technique (e.g. footnote 1), the criteria obtained are too impractical to be applied for each case. For all practical purposes, however, a simple rule of thumb is usually sufficient to consider. If (1) can be so ordered that

$$
a_{i j} \gg\left(a_{i 1}, a_{i 2}, \cdots, a_{i, j-1}, a_{i, j+1}, \cdots, a_{i n}\right) \quad j=1,2, \cdots, n,
$$

it can be safely concluded, in general, that the Iterative technique will converge.

It is the aim of this note to demonstrate how conventional techniques can be practicably utilized to transform (1) into an equivalent system which satisfies the conditions (2), thereby enabling the computer to take full advantage of the digital type of solution possible by the Iterative technique. It is the opinion of the author that in general, it is more practical computationally to regard techniques involving "rounding off" errors such as analogue computers, Crout's Method, ${ }^{2}$ etc., as auxiliary tools for obtaining an equivalent system satisfying (2) rather than methods for obtaining a final solution directly.

*Received Dec. 30, 1949.

${ }^{1} \mathrm{H}$. Hotelling, Some new methods of matrix calculation, Ann. Math. Stat. 14, 1-34 (1943).

2P. D. Crout, $A$ short method for evaluating determinants and solving systems of linear equations with real or complex coefficients, Presented at A.I.E.E. Summer Convention, 1941. Published by A.I.E. 1941. 